\title{
A organização de uma revisão de literatura por meio da Tree of Science (Árvore da Ciência): um exemplo sobre a avaliação da pós-graduação ${ }^{1}$
}

\author{
The review of literature through the Tree of Science: \\ an example with Postgraduate assessment
}

\author{
Roberto Patrus ${ }^{1}$ \\ ${ }^{1}$ Pontifícia Universidade Católica de Minas Gerais | Programa de Pós-graduação em \\ Administração | Departamento de Administração \\ Belo Horizonte | MG | Brasil. Contato: robertopatrus@ pucminas.br \\ http://orcid.org/0000-0001-7511-4107 \\ Vinícius Tolentino Oliveira e Silva ${ }^{2}$ \\ ${ }^{2}$ Pontifícia Universidade Católica de Minas Gerais | Programa de Pós-graduação em \\ Administração | Departamento de Administração \\ Belo Horizonte | MG | Brasil. Contato: vinycius01 @ gmail.com \\ http://orcid.org/0000-0002-0437-6211
}

Resumo: O advento da busca por artigos por meio da Web tornou a pesquisa bibliográfica mais rápida e econômica. Difícil, no entanto, é organizar a enorme quantidade de papers que retornam da pesquisa. Embora haja na literatura inúmeros artigos sobre os métodos e técnicas de pesquisa, poucos são os trabalhos que abordam as técnicas de revisão de literatura. Tendo em vista tal lacuna teórica e a dificuldade prática dos estudantes de mestrado e doutorado em organizar o referencial teórico de seus trabalhos acadêmicos, objetivamos com este artigo apresentar uma ferramenta que tem se mostrado útil para a organização da revisão de literatura de teses e dissertações: a Tree of Science (ToS), Árvore da Ciência. Com este recurso, os pesquisadores realizam buscas nas bases de dados da Web Of Science (WOS), criam um arquivo de texto com as referências selecionadas e o submete para a montagem de uma árvore, que classifica as referências encontradas em três níveis, utilizando conceitos da teoria dos grafos, por meio da análise das redes de citações: a raiz, que reúne os artigos seminais; o tronco, que agrega artigos estruturadores; e as folhas, que indica perspectivas de pesquisa, em geral artigos mais recentes. Como forma de trazer um exemplo do uso da ferramenta ToS, realizamos uma busca pelos termos “Avaliação" e "Pós-graduação". Nossa revisão organizou quatro perspectivas: Avaliação da pósgraduação / Capes, Produtivismo, Avaliação educacional e Produção cientifica. Por meio desse exemplo, o artigo demonstra a utilidade da ToS para a organização da pesquisa bibliográfica.

Palavras-chave: Revisão de literatura. Tree of Science. Avaliação da pós-graduação.

Abstract: The advent of the search for articles through the Web has made bibliographic research faster and more economical. Difficult, however, is to organize the huge amount of papers that return from the search. Although there are innumerable articles on the methods and techniques of research, there are few papers about techniques of literature review. Faced with this theoretical gap and the practical difficulty of master's and doctoral students in organizing the literature review in their academic works, this paper aims to present a useful tool for the organization of literature review of theses and dissertations: the Tree of Science (ToS). With this feature, researchers search the Web of Science (WOS) databases and create a text file with the selected references. Then, by submitting this file to ToS, the site constructs a tree, using graph theory concepts, through the analysis of the citation networks. The tree classifies the references found in three levels: root, which gathers the seminal articles; trunk, which aggregates structuring articles; and leaves, which indicate the recent articles. It is up to the researcher to design the branches, that means the approaches in which the articles are located. As a way to bring an example of

\footnotetext{
${ }^{1}$ Os autores agradecem o apoio da Capes e da FAPEMIG.
} 
the use of the ToS tool, we search for the terms "Evaluation" and "Postgraduate". Our review organized four perspectives (branches): Postgraduate Evaluation / Capes, Productivism, Educational Evaluation, and Scientific Production. Through this example, the article demonstrates the usefulness of ToS for the organization of bibliographic research.

Key words: Literature review. Tree of Science. Postgraduate assessment.

DOI: http://dx.doi.org/10.1590/S1414-40772019000100005

Recebido em: 12 de maio de 2017

Aprovado em: 19 de setembro 2018

Este é um artigo publicado em acesso aberto (Open Access) sob a licença Creative Commons Attribution Non-Commercial, que permite uso, distribuição e reprodução em qualquer meio, sem restrições desde que sem fins comerciais e que o trabalho original seja corretamente citado. https://creativecommons.org/licenses/by-nc/4.0/

\section{Introdução}

A partir dos anos 1990, com avanço da informatização, as revistas passaram a ser dispostas na web. Com as revistas eletrônicas, a busca por artigos se tornou mais simples. Com o surgimento das Bases de Dados, a reunião de diferentes periódicos em um mesmo sítio propiciou economia de tempo para o pesquisador, que não mais tem de ir a cada revista para buscar os artigos de seu interesse.

Atualmente existem diversas bases de dados contendo revistas e artigos. Uma das maiores bases de dados é a Web of Science (WOS) que disponibiliza acesso a mais de 12.000 títulos de periódicos (CAPES, 2017). A WOS, anteriormente conhecida como "Web of Knowledge”, permite a indexação de citações científicas e o cálculo do Fator de Impacto de cada periódico por meio de recursos bibliométricos. Originalmente produzido pelo Instituto de Informação Científica (ISI), agora mantido pela Clarivate Analytics (anteriormente propriedade da Thomson Reuters), a WOS fornece uma busca bem abrangente de referências. Ela dá acesso a vários bancos de dados que permitem a exploração em profundidade de sub-campos especializados dentro de uma disciplina acadêmica ou científica (DRAKE, 2004). Ela permite acesso a referências e resumos em todas as áreas do conhecimento. Por meio da WOS, estão disponíveis ferramentas para análise de citações, referências, índice h e outras análises bibliométricas.

Antes do advento da tecnologia digital, a busca por um artigo era feita em bibliotecas e a fotocópia do artigo enviada pelo correio, semanas depois. Agora ficou mais fácil. Difícil, no entanto, é organizar a enorme quantidade de papers que retornam da pesquisa para decidir o que ler e o que não ler. Embora haja na literatura inúmeros artigos sobre os métodos e técnicas 
de pesquisa, poucos são os trabalhos que abordam as técnicas de revisão de literatura ou pesquisa bibliográfica.

O resultado dessa desatenção ao processo de revisão de literatura tornou célebre o artigo de Alves (1992), revisado e publicado posteriormente (ALVES-MAZZOTTI, 2006) no livro organizado por Bianchetti e Machado (2006) com a constatação da autora de que, infelizmente, “os problemas continuam os mesmos" (ALVES-MAZZOTTI, 2006, p. 25). Onze anos depois, constatamos que "os tipos de revisão a serem evitados" (ALVES-MAZZOTTI, 2006, p. 26) continuam se repetindo nas teses e dissertações. Entre eles, recordamos alguns: a "Summa", revisão que ilusoriamente tenta esgotar o assunto, desde o período mais antigo; o "Patchwork", aquela que apresenta uma colagem de conceitos, pesquisas e afirmações de diversos autores, sem um fio condutor capaz de guiar o leitor; o "Suspense", roteiro pelo qual o tema de estudo só aparece nas páginas finais do capítulo; o "Rococó", em que conceituações teóricas rebuscadas tentam dar elegância a dados irrelevantes; o "Caderno B", o texto leve que trata o tema de modo ligeiro, geralmente a partir de livros didáticos e handbooks; "o Ventríloco", revisão em que o autor só fala pelos autores citados, sem análises críticas e tomadas de posição.

Tendo em vista, por um lado, a dificuldade prática dos estudantes de mestrado e doutorado em organizar o referencial teórico de seus trabalhos acadêmicos e, por outro, a lacuna teórica na produção de artigos sobre esse assunto, objetivamos com este artigo apresentar uma ferramenta que tem se mostrado útil para a organização da revisão de literatura de teses e dissertações: a Tree of Science (ToS), Árvore da Ciência. A ToS é uma é uma ferramenta baseada na web que utiliza algoritmos de rede para otimizar a pesquisa e seleção de artigos científicos. Com este recurso, os pesquisadores realizam buscas nas bases de dados da WOS, criam um arquivo de texto com as referências selecionadas e os submete para a montagem de uma árvore, que classifica as referências encontradas em três níveis: a raiz, que reúne os artigos seminais; o tronco, que agrega artigos estruturadores; e as folhas, que indica perspectivas de pesquisa, em geral artigos mais recentes (TREE OF SCIENCE, 2017). Além da forma gráfica de árvore, é possível elencar os artigos em arquivo Excel, no formato de campos separados por vírgula (.csv).

A classificação das referências em artigos seminais, estruturadores e mais recentes obedece a técnicas bibliométricas utilizando conceitos da teoria dos grafos, por meio da análise das redes de citações. Três indicadores são utilizados: grau de entrada, intermediação e grau de saída. Os artigos com grau de entrada alto e saída zero são os denominados seminais. Os artigos com um grau de intermediação alto são os chamados troncos. Aqueles que tem um grau de saída alto e um grau de entrada zero são denominados folhas (ROBLEDO; OSORIO; LOPEZ, 2015). 
A partir do conhecimento prévio do pesquisador, ou do grupo de pesquisadores, considerando a participação do orientador, é possível construir os galhos, que são as perspectivas teóricas que agrupam as folhas.

O uso da ToS vem se mostrando muito útil nos trabalhos acadêmicos do grupo de pesquisa do qual fazemos parte. Podemos de fato constatar que seu slogan é de fato verdadeiro: "search less, research more". A economia de tempo com a busca por artigos tem propiciado maior disponibilidade para a sua leitura e para um referencial teórico diferente dos tipos elencados por Alves (1992) e recordados aqui.

Como forma de trazer um exemplo do uso da ferramenta ToS, realizamos uma busca pelos termos "Avaliação" e "Pós-graduação" (avaliação and pós-graduação) na base de dados WOS no SciELO Citation Index. A opção por limitar a pesquisa ao Scielo teve como objetivo restringir a busca a publicações de autores brasileiros, visto que a dissertação de mestrado a ser realizada por um dos autores tem como tema a avaliação da pós-graduação brasileira coordenada pela Capes. A partir da pesquisa na WOS, foi criada a árvore da ciência com artigos seminais (raiz), estruturadores (tronco) e os derivados das perspectivas por nós determinadas (folhas). Nossa revisão organizou quatro perspectivas: (1) Avaliação de Programas de Pósgraduação / Capes, (2) Produtivismo, (3) Avaliação educacional e (4) Produção cientifica.

A próxima seção deste artigo descreve o processo de seleção de artigos na WOS e a criação da árvore da ciência no ToS. A terceira seção explica as diferentes perspectivas criadas pelos autores a partir da base dados da WOS, e, na última seção, fazemos as considerações finais.

\section{Um exemplo do uso da ferramenta ToS}

Por interesse dos pesquisadores, foram definidos os termos da busca: "avaliação" e “pós-graduação". Em seguida, foi realizada uma pesquisa na base de dados da WOS no Scielo Citation Index. Como já dito, a busca pelo termo em português é estratégica para esta pesquisa, dado o interesse em encontrar evidências sobre a avaliação da pós-graduação no Brasil, especificamente a Avaliação Capes de Programas de Pós-graduação. A sintaxe de busca adotou o seguinte modelo: pesquisa por tópico "avaliação" e (and) pesquisa por tópico "pósgraduação", refinado por tipos de documento "Research article" nas categorias da Scielo: "Education Education research or Management" em todos os anos no índice "Scielo" (Figura 1). 
Figura 1 - Configuração da busca

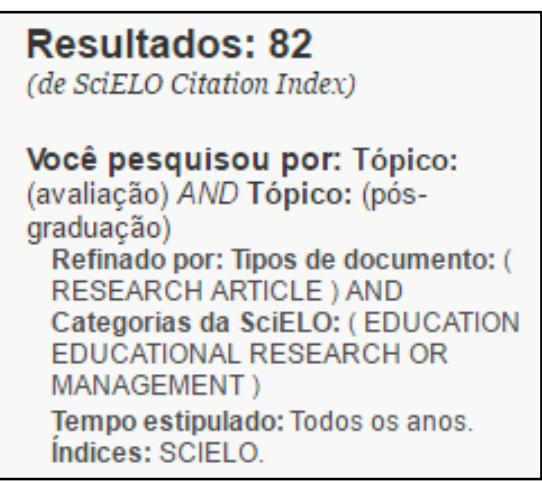

Fonte: Dados da busca, a partir da base de dados WOS.

Realizada a busca na WOS, foram retornados 82 artigos, um número bem inferior ao de outras pesquisas realizadas por nós. São poucas as investigações sobre o tema no Brasil. Para gerar a árvore da ciência no ToS, é necessária a criação de um arquivo texto na WOS contendo "registro completo e referências citadas". Este arquivo é submetido na ToS em "New search" a fim de gerar a árvore da ciência. A árvore fica armazenada em "Uploads” e poderá ser consultada quando necessário. Esta é configurada na forma padrão, em formato de uma lista onde os artigos são antecedidos de pequenos círculos amarelos, marrons ou verdes. Os círculos amarelos são as referências seminais, os marrons são os estruturadores, e os verdes são os mais recentes.

Na figura 2, são apresentados os resultados em forma gráfica (árvore), organizado desta forma, a fim de exibir melhor os indicadores acima.

Figura 2 - Resultado em forma de árvore da ciência

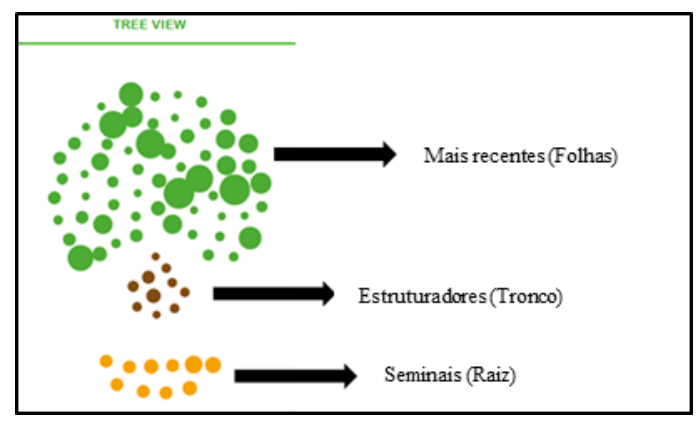

Fonte: Elaborado pelos autores.

$\mathrm{Na}$ árvore da ciência, cada círculo representa uma publicação, podendo ser livro, artigo ou um documento. Ao clicar em cada um desses círculos, é exibida a respectiva referência. No caso de artigo, aparecem o autor, ano, volume e página da revista em que o artigo foi publicado. 
Para alguns artigos existe a possibilidade de acessá-lo via link pelo Digital Object Identifier (DOI). Para documentos, como o Diário Oficial da União, Documentos Capes ou mesmo anais de congressos, os dados da tabela não permitem a identificação direta, exigindo do pesquisador que os busque nas referências bibliográficas dos artigos encontrados. Para este artigo, em função do seu objetivo, limitamos o exemplo aos artigos.

Das dez referências elencadas como seminais, raiz da árvore, cinco são documentos, como o Diário Oficial da União, de 2001, o Documento Capes da área de Educação 2004, um documento denominado Anais, de 2009, que não conseguimos identificar, o V Plano Nacional de Educação, de 2004, e novamente um Diário Oficial da República Federativa do Brasil, de 1966. Os artigos publicados em periódicos são listados no Quadro 1, a seguir.

Quadro 1 - A árvore do conhecimento sobre a Avaliação e pós-graduação: artigos seminais (raiz)

\begin{tabular}{|c|}
\hline Ferraro (2005) \\
\hline Godoi e Xavier (2012) \\
\hline Horta e Moraes (2005) \\
\hline Kuenzer e Moraes (2005) \\
\hline Machado e Bianchetti (2011) \\
\hline
\end{tabular}

Fonte: Elaborado pelos autores.

O tronco da árvore da ciência sobre a avaliação da pós-graduação recebeu 10 referências, entre eles dois documentos, um do Conselho Técnico Científico da Capes, denominado "Reestruturação do Qualis", de 2009 e o Documento da área de Educação, também da Capes, de 2004, curiosamente também classificado como artigo seminal. Os outros 8 documentos são artigos, indicados no Quadro 2.

Quadro 2 - A árvore do conhecimento sobre a Avaliação e pós-graduação: artigos estruturais (tronco)

\begin{tabular}{|c|}
\hline Córdova, Gusso e Luna (1986) \\
\hline Dias e Rorato (2014) \\
\hline Gurgel e Leite (2007) \\
\hline Lüdke (2005) \\
\hline Maccari, Almeida e Rodrigues (2009) \\
\hline Muñoz e Alonso (2015) \\
\hline Sinay et. al (2013) \\
\hline Verhine (2008) \\
\hline
\end{tabular}

Fonte: Elaborado pelos autores.

A copa da árvore da ciência, suas folhas, reuniu na busca do ToS, 60 referências, todas elas artigos científicos. Do total, apenas três artigos não ofereciam acesso pelo link disponível 
no ToS. Destes três artigos, apenas um não foi localizado em buscas no Scielo, no Google, no Currículo Lattes e na Web em geral. Os 59 artigos encontrados estão citados no Quadro 3, a seguir.

Quadro 3 - A árvore do conhecimento sobre a Avaliação e pós-graduação: artigos mais recentes (folhas)

\begin{tabular}{|c|c|}
\hline Almeida e Borges (2007) & Mello, Crubellate e Rossoni (2010) \\
\hline Apio e Silvino (2013) & Mello, Crubellate e Rossoni (2009) \\
\hline Bispo e Costa (2016) & Moreira (2009) \\
\hline Calvosa, Repossi e Castro (2011) & Moreira e Velho (2008) \\
\hline Canhada e Bulgacov (2011) & Moreira e Velho (2012) \\
\hline Castro (2011) & Muñoz e Alonso (2015) \\
\hline Castro e Porto (2012) & Nosella (2010) \\
\hline Cirani, Silva e Campanario (2012) & Patrus, Dantas e Shigaki (2015) \\
\hline Dias e Rorato (2014) & Peixoto (2011) \\
\hline Embiruçu, Fontes e Almeida (2010) & Pezzi e Steil (2009) \\
\hline Festinalli (2005) & Pucci (2007) \\
\hline Fialho e Hetkowski (2017) & Ramalho e Madeira (2005) \\
\hline Fischer (2007) & Ramos e Velho (2013) \\
\hline Francisco et al. (2016) & Rauen e Filho (2016) \\
\hline Gurgel e Leite (2007) & Rego (2014) \\
\hline Hortale e Koifman (2007) & Rocha (2006) \\
\hline Hostins (2013) & Rosa e Lopes (2016) \\
\hline Ikeda e Bacellar (2008) & Sanches e Sanches (2011) \\
\hline Kuenzer e Moraes (2005) & Silva e Nakano (2012) \\
\hline Kuhlmann (2015) & Sinay et al. (2013) \\
\hline Maccari, Riccio e Martins (2013) & Sobral (2009) \\
\hline Maccari e Nishimura (2014) & Souza e Reinert (2012) \\
\hline Maccari, Almeida, Riccio e Alejandro (2014) & Trevisan, Devechi e Dias (2013) \\
\hline Macedo (2015) & Velloso (2004) \\
\hline Macedo e Sousa (2010) & Vianna e Giffhorn (2011) \\
\hline Machado-da-Silva (2003) & Vidor, Fogliatto e Caten (2013) \\
\hline Marin et al. (2010) & Vilaça e Palma (2015) \\
\hline Marquezine e Tramontina (2006) & Werle, (2011) \\
\hline \multirow[t]{2}{*}{ Martins e Sousa (2012) } & Werle (2014) \\
\hline & Wood Jr. e Costa (2015) \\
\hline
\end{tabular}

Fonte: Elaborado pelos autores.

A partir dessa busca, está reunido o material bibliográfico que serve como ponto de partida para a revisão teórica pertinente à pesquisa. Observamos que algumas referências se repetem, como o caso de Kuenzer e Moraes (2005), que está na raiz e na folha da árvore, assim como Dias e Rorato (2014), que está no tronco e na folha, mesmo caso de Gurgel e Leite (2007) e Muñoz e Alonso (2015). De posse dos artigos, resta organizar a estrutura do referencial teórico 
do trabalho, que vai depender de como o pesquisador analisa os seus conteúdos e classifica as perspectivas teóricas presentes na literatura pesquisada.

\section{Alternativas de estruturação da revisão bibliográfica}

Existem várias opções de organização da pesquisa bibliográfica, tanto em termos de forma como de conteúdo. Apresentamos inicialmente a organização formal. No item seguinte, descrevemos a organização em termos de conteúdo, a partir das perspectivas criadas pelos autores na revisão de literatura do nosso exemplo, a avaliação da pós-graduação.

\subsection{Critérios formais de organização da revisão bibliográfica}

A classificação das referências encontradas na busca pode ser feita pelo site do Web of Science. Em termos de forma, os critérios podem ser geográficos, por idioma da publicação, instituição de origem, distribuição dos artigos por ano de publicação, e, por fim, os veículos responsáveis, a revista ou a editora.

A partir do critério geográfico, no nosso exemplo já era esperado que as referências da busca não estivessem distribuídas de forma homogênea. Do total de 82 referências, 69 foram identificadas como brasileiras, sendo que o restante se distribuiu por Espanha (3 publicações), Argentina (2 publicações) e Colômbia, Cuba, Inglaterra, México, Peru, Portugal, Timor Leste e Estados Unidos (com uma referência cada).

A distribuição anterior confirma que a maior parte dos artigos foi escrita no idioma português, 73 , o que corresponde a um percentual de $89,03 \%$. Nove trabalhos foram escritos em espanhol, representando 10,97\%.

Como a maioria dos artigos são de nacionalidade brasileira, existe uma grande concentração destes artigos publicados por instituições de ensino superior brasileiras. Dos 82 artigos encontrados, a Universidade de São Paulo (USP) é a instituição que que mais publicou sobre o tema pesquisado, são 9 artigos (10,97\%), seguida da Universidade Federal do Rio Grande do Sul (UFRS), com 5 artigos (6,09\%). A Figura 3 ilustra a distribuição de artigos por instituição de ensino superior. 
Figura 3 - Distribuição de artigos por instituição

\begin{tabular}{|c|c|c|c|}
\hline Campo: Instituiçôes & Contagem do registro & $\%$ de 82 & Gráfico de barras \\
\hline UNIVERSIDADE DE SAO PAULO & 9 & $10.976 \%$ & [ \\
\hline UNIVERSIDADE FEDERAL DO RIO GRANDE DO SUL & 5 & $6.098 \%$ & - \\
\hline FUNDACAO OSWALDO CRUZ & 4 & $4.878 \%$ & I \\
\hline UNIVERSIDADE DO ESTADO DO RIO DE JANEIRO & 4 & $4.878 \%$ & I \\
\hline UNIVERSIDADE FEDERAL DE SANTA CATARINA & 4 & $4.878 \%$ & I \\
\hline UNIVERSIDADE FEDERAL DO PARANA & 4 & $4.878 \%$ & I \\
\hline UNIVERSIDADE NOVE DE JULHO & 4 & $4.878 \%$ & I \\
\hline UNIVERSIDADE ESTADUAL DE CAMPINAS & 3 & $3.659 \%$ & I \\
\hline UNIVERSIDADE FEDERAL DE SAO CARLOS & 3 & $3.659 \%$ & I \\
\hline UNIVERSIDADE FEDERAL DO RIO DE JANEIRO & 3 & $3.659 \%$ & I \\
\hline
\end{tabular}

Fonte: Dados da busca, a partir da base de dados WOS.

Outra análise importante que a WOS provê é a quantidade de referências sobre o termo pesquisado por ano. No ano de 2011, foram publicados 10, seguido de 2012 com 9 referências, e 2013 com 8. Os demais anos possuem uma distribuição homogênea, variando no máximo em um ponto. A Figura 4 ilustra a distribuição de artigos do tema pesquisado por ano.

Figura 4 - Distribuição de artigos por ano -

\begin{tabular}{|r|c|c|c|}
\hline Campo: Anos de publicação & Contagem do registro & $\%$ de 82 & Gráfico de barras \\
\hline 2011 & 10 & $12.195 \%$ & \\
2012 & 9 & $10.976 \%$ & $\mathbf{0}$ \\
2013 & 8 & $9.756 \%$ & $=$ \\
2007 & 7 & $8.537 \%$ & $=$ \\
2014 & 7 & $8.537 \%$ & $=$ \\
2015 & 7 & $8.537 \%$ & $\square$ \\
2009 & 6 & $7.317 \%$ & $=$ \\
2010 & 6 & $7.317 \%$ & $\square$ \\
2005 & 5 & $6.098 \%$ & \\
2016 & 5 & $6.098 \%$ & \\
\hline
\end{tabular}

Fonte: Dados da busca, a partir da base de dados WOS.

Uma outra informação que vale ressaltar é a distribuição de artigos por veículos de informação. A “Revista Avaliação da Educação Superior” (RAES) é a que mais publica sobre o termo pesquisado, com 17 itens, um total de 20,73\%, seguida da "Ensaio: Avaliação e Políticas Públicas em Educação" e "Revista Brasileira de Educação", ambas com 9 artigos, representando um percentual de $10,97 \%$ cada uma. As demais revistas possuem o mesmo número de artigos, variando em um ponto (Figura 5). 
Figura 5 - Distribuição de artigos veículos de informação

\begin{tabular}{|c|c|c|c|}
\hline Campo: Titulos da fonte & Contagem do registro & $\%$ de 82 & Gráfico de barras \\
\hline $\begin{array}{l}\text { AVALIACAO REVISTA DA AVALIACAO DA EDUCACAO SUPERIOR } \\
\text { CAMPINAS }\end{array}$ & 17 & $20.732 \%$ & - \\
\hline ENSAIO AVALIACAO E POLITICAS PUBLICAS EM EDUCACAO & 9 & $10.976 \%$ & a \\
\hline REVISTA BRASILEIRA DE EDUCACAO & 9 & $10.976 \%$ & 口 \\
\hline CADERNOS DE PESQUISA & 4 & $4.878 \%$ & I \\
\hline INTERFACE COMUNICACAO SAUDE EDUCACAO & 4 & $4.878 \%$ & I \\
\hline CADERNOS EBAPE BR & 3 & $3.659 \%$ & I \\
\hline EDUCACAO E PESQUISA & 3 & $3.659 \%$ & I \\
\hline EDUCACAO SOCIEDADE & 3 & $3.659 \%$ & I \\
\hline ORGANIZACOES SOCIEDADE & 3 & $3.659 \%$ & I \\
\hline RAM REVISTA DE ADMINISTRACAO MACKENZIE & 3 & $3.659 \%$ & I \\
\hline
\end{tabular}

Fonte: Dados da busca, a partir da base de dados WOS (WEB OF SCIENCE, 2017)

Muitas revisões de literatura se resumem a descrever aspectos formais como os apontados anteriormente, o que pouco acrescenta à organização do estado da arte da pesquisa sobre o tema. Os artigos sequer precisam ser lidos para que essa análise seja feita. E a base de dados pode fazer isso para o pesquisador, como foi o caso da WOS. Outra forma de organizar a revisão de literatura é a partir do seu conteúdo. Esta se mostra bem mais importante. É o que veremos a seguir.

3.2 Critérios de organização da revisão bibliográfica baseados no conteúdo dos artigos

Em termos de conteúdo, o maior trabalho consiste em conceber os galhos (ou ramos) onde os artigos da árvore se situam. Chamamos os galhos de perspectivas. Essa análise exige a leitura dos artigos e a sua sistematização já possibilita que o autor coloque a sua marca na revisão bibliográfica, na medida em que é ele que classifica os artigos e os organiza em termos de conteúdo, abordagem ou perspectiva teórica.

Na revisão de literatura que tomamos como exemplo, identificamos quatro perspectivas a partir da leitura dos artigos, a saber: Avaliação da pós-graduação / Capes; Produção científica; Produtivismo acadêmico (que também pode ser chamado de cultura da performatividade); e Avaliação educacional. A perspectiva que concentrou maior número de artigos foi a Avaliação da Pós-graduação / Capes. A partir da leitura dos artigos, vemos que a perspectiva Produção Científica é um corolário da primeira perspectiva, ao passo que o Produtivismo Acadêmico é um efeito colateral deste.

A perspectiva Avaliação educacional, por outro lado, aborda temas desde avaliação docente até avaliação institucional da educação, passando por avaliação da aprendizagem. Tal organização dos artigos ajuda o pesquisador a eleger o fio condutor da revisão teórica que está empreendendo, seja para limitar a sua análise, seja para necessariamente tratar do assunto. Por exemplo, se seu foco na pesquisa é a produção acadêmica, a revisão da literatura pode levá-lo a excluir o tema do produtivismo ou, ao contrário, pode exigir que ele o aborde como efeito 
perverso da avaliação da produção acadêmica. Essa decisão compete ao pesquisador e seu grupo de pesquisa, de acordo com suas prioridades, seus limites, condições de trabalho, etc.

Vale a pena observar que, nos artigos seminais, classificamos três artigos na perspectiva da Avaliação da Pós-graduação / Capes, um artigo na perspectiva produção científica e um artigo na perspectiva Produtivismo Acadêmico. Apenas a perspectiva avaliação educacional não teve artigo de periódico entre as referências de raiz. Na categoria "tronco", de artigos estruturadores, não classificamos nenhum artigo apenas na perspectiva produção científica (Quadro 4).

Quadro 4 - Classificação dos artigos da árvore do conhecimento em perspectivas

\begin{tabular}{|c|c|c|c|c|}
\hline & $\begin{array}{l}\text { Avaliação da pós- } \\
\text { graduação / Capes }\end{array}$ & $\begin{array}{l}\text { Produção } \\
\text { científica }\end{array}$ & $\begin{array}{l}\text { Produtivismo } \\
\text { acadêmico }\end{array}$ & $\begin{array}{c}\text { Avaliação } \\
\text { Educacional }\end{array}$ \\
\hline \multirow{19}{*}{$\begin{array}{l}\mathbf{F} \\
\mathbf{O} \\
\mathbf{L} \\
\mathbf{H} \\
\mathbf{A} \\
\mathbf{S}\end{array}$} & Almeida e Borges (2007) & $\begin{array}{l}\text { Calvosa, Repossi } \\
\text { e Castro (2011) }\end{array}$ & $\begin{array}{c}\text { Bispo e Costa } \\
(2016)\end{array}$ & $\begin{array}{l}\text { Moreira e Velho } \\
(2012)\end{array}$ \\
\hline & $\begin{array}{c}\text { Canhada e Bulgacov } \\
\text { (2011) }\end{array}$ & $\begin{array}{c}\text { Castro e Porto } \\
\text { (2012) }\end{array}$ & Macedo (2015) & Werle (2011) \\
\hline & Dias e Rorato (2014) & $\begin{array}{l}\text { Mello, Crubellate } \\
\text { e Rossoni (2009) }\end{array}$ & $\begin{array}{c}\text { Macedo e } \\
\text { Sousa (2010) }\end{array}$ & $\begin{array}{c}\text { Francisco et al. } \\
(2016)\end{array}$ \\
\hline & Fialho e Hetkowski (2017) & $\begin{array}{l}\text { Souza e Reinert } \\
\quad(2012)\end{array}$ & $\begin{array}{l}\text { Patrus, Dantas } \\
\text { e Shigaki } \\
(2015)\end{array}$ & Velloso (2004) \\
\hline & $\begin{array}{l}\text { Maccari, Riccio e Martins } \\
\text { (2013) }\end{array}$ & Sobral (2009) & Rego (2014) & Marin et al. (2010) \\
\hline & $\begin{array}{c}\text { Maccari e Nishimura } \\
(2014)\end{array}$ & $\begin{array}{l}\text { Filho e Martins } \\
\text { (2006) }\end{array}$ & $\begin{array}{c}\text { Vilaça e Palma } \\
(2015)\end{array}$ & $\begin{array}{l}\text { Embiruçu, Fontes e } \\
\text { Almeida (2010) }\end{array}$ \\
\hline & $\begin{array}{l}\text { Mello, Crubellate e } \\
\text { Rossoni (2010) }\end{array}$ & Fisher (2007) & $\begin{array}{l}\text { Kuhlmann } \\
\text { (2015) }\end{array}$ & $\begin{array}{c}\text { Ikeda e Bacellar } \\
\text { (2008) }\end{array}$ \\
\hline & Moreira e Velho (2008) & $\begin{array}{l}\text { Martins e Sousa } \\
\quad(2011)\end{array}$ & Moreira (2009) & $\begin{array}{l}\text { Rauen \& Filho } \\
\text { (2016) }\end{array}$ \\
\hline & Sanches e Sanches (2011) & $\begin{array}{c}\text { Wood Jr. e Costa } \\
(2015)\end{array}$ & & Pucci (2007) \\
\hline & $\begin{array}{l}\text { Maccari, Almeida, Riccio } \\
\text { e Alejandro (2014) }\end{array}$ & $\begin{array}{l}\text { Marquezine e } \\
\text { Tramontina } \\
\text { (2006) }\end{array}$ & & Peixoto (2011) \\
\hline & Rocha (2006) & $\begin{array}{c}\text { Silva \& Nakano } \\
(2012)\end{array}$ & & $\begin{array}{l}\text { Vidor, Fogliatto e } \\
\text { Caten (2013) }\end{array}$ \\
\hline & Vianna e Giffhorn (2011) & Nosella (2010) & & $\begin{array}{l}\text { Rosa e Lopes } \\
\text { (2016) }\end{array}$ \\
\hline & Ramos e Velho (2013) & & & Castro (2011) \\
\hline & Ramalho e Madeira (2005) & & & $\begin{array}{l}\text { Apio e Silvino } \\
\text { (2013) }\end{array}$ \\
\hline & $\begin{array}{l}\text { Trevisan, Devechi e Dias } \\
\text { (2013) }\end{array}$ & & & Werle (2014) \\
\hline & Machado-da-Silva (2003) & & & \\
\hline & Festinalli (2005) & & & \\
\hline & Fischer (2007) & & & \\
\hline & Pezzi e Steil (2009) & & & \\
\hline \multirow{3}{*}{$\begin{array}{l}\mathbf{T} \\
\mathbf{R}\end{array}$} & & & & \\
\hline & Verhine (2008) & & $\begin{array}{c}\text { Muñoz e } \\
\text { Alonso (2015) }\end{array}$ & $\begin{array}{l}\text { Gurgel e Leite } \\
\text { (2007) }\end{array}$ \\
\hline & Lüdke (2005) & & & \\
\hline
\end{tabular}




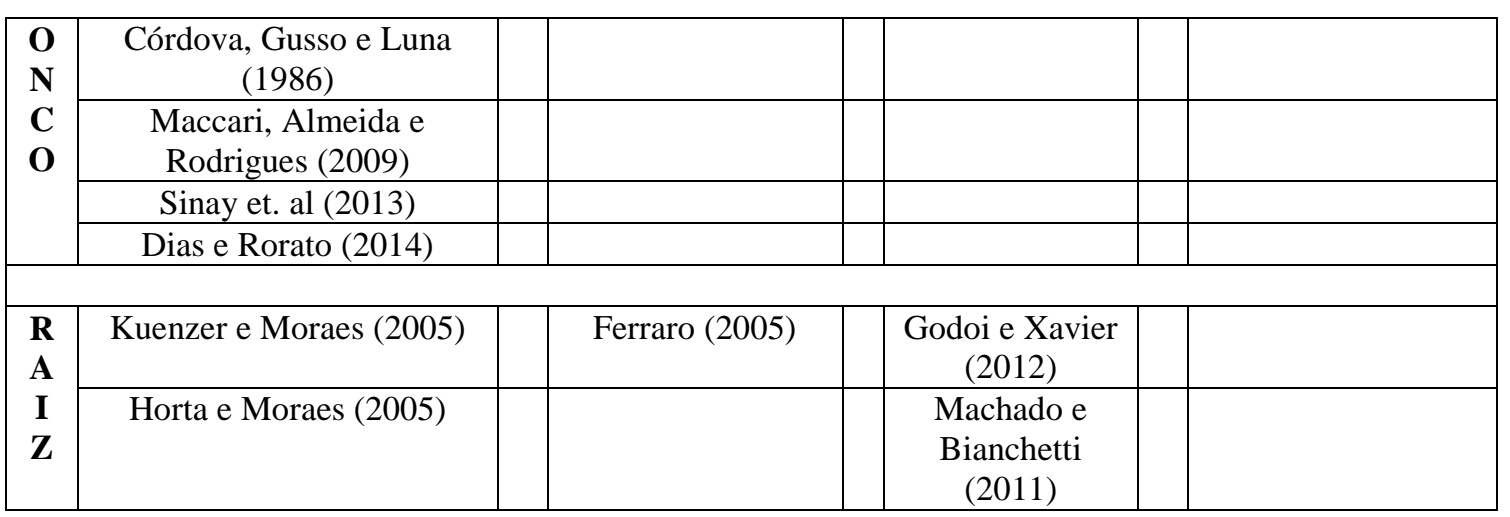

Fonte: Elaborado pelos autores (TREE OF SCIENCE, 2017)

Vale a ressalva de que limitamos esta classificação a artigos de periódicos, excluindo os documentos de área, diários oficiais e artigos de anais de congressos.

\section{Considerações finais}

Não há projeto de pesquisa consistente sem uma boa revisão de literatura. Não há pesquisa sem a leitura da revisão de literatura. Não há produções resultantes da pesquisa, como artigos científicos, sem referências bibliográficas. Seja na formulação de um problema de pesquisa, seja na concepção do projeto, seja durante a análise dos dados, seja na produção dos artigos resultantes da investigação, a leitura se faz presente de forma necessária e imprescindível. Em nosso grupo de pesquisa, costumamos repetir que "sem input não há output". Se o produto da atividade de pesquisa é o artigo científico, um dos seus inputs fundamentais é a leitura.

Essa máxima foi muito bem traduzida por Rössing (2002), quando pergunta: "se não leem ou leem pouco, como esperar que escrevam?" (p. 68). Entretanto, diante de tão numerosas e vastas fontes de pesquisa, tão importante quanto ler é saber o que não ler. Para definir as leituras que devem ser dispensadas, é preciso saber definir bem o problema de pesquisa. Definir, etimologicamente, significa colocar fim, demarcar as fronteiras, marcar os limites, ou seja, de-limitar. Pensamos que a ferramenta do Tree of Science, elaborada a partir da busca na base de dados do Web of Science, é uma ótima estratégia para dar ao texto da revisão teórica o seu fio condutor.

Não queremos com isso dizer que a pesquisa deva se limitar a essa base de dados, mas que é uma possibilidade para permitir ao pesquisador, com a leitura dos textos selecionados, elaborar o fio condutor de sua revisão bibliográfica. Tal fio condutor permitirá que a pesquisa 
em outras bases de dados como Scopus, por exemplo, seja feita a partir das perspectivas propostas a partir da pesquisa do WOS.

Como principal contribuição teórica do artigo, pensamos oferecer aqui a possibilidade de a revisão da literatura não ser vítima dos problemas tão bem levantados por Alves (1992) a respeito das revisões bibliográficas. Ao contrário, poderá constituir-se em uma verdadeira construção teórica, na medida em que permite ao pesquisador organizar as perspectivas a partir das quais pretende abordar o tema do seu trabalho acadêmico. A elaboração das perspectivas depende do conhecimento prévio do pesquisador. Em caso de trabalhos de mestrado, ou mesmo, de doutorado, a participação do orientador se faz necessária para garantir melhor classificação das publicações encontradas na busca.

Um dos limites para que a pesquisa possa ser realizada é o acesso à base de dados do WOS. Ele está disponível na Plataforma Periódicos, da Capes, mas não para todas as Instituições de Ensino Superior. Trata-se de uma base de dados que exige uso de senha, disponibilizada pela instituição responsável pela assinatura. Já o acesso ao Tree of Science depende apenas de convite de uma pessoa que tenha acesso. Os autores desse artigo se colocam a disposição para fazê-lo a todos os que lhes comunicarem o seu interesse.

\section{Referências}

ALMEIDA, Nival Nunes de; BORGES, Mário Neto. A pós-graduação em engenharia no Brasil: uma perspectiva histórica no âmbito das políticas públicas. Ensaio, Rio de Janeiro, v. 15, n. 56, p. 323-339, set. 2007. Doi: http://dx.doi.org/10.1590/S0104-40362007000300002. Disponível em: http://www.scielo.br/scielo.php?script=sci_arttext\&pid=S0104$40362007000300002 \& \operatorname{lng}=$ pt\&tlng=pt. Acesso em: 10 maio 2017.

ALVES, Alda Judith. Revisões bibliográficas em teses e dissertações: meus tipos inesquecíveis. Cadernos de Pesquisa-Fundação Carlos Chagas, São Paulo, p. 53-61, 1992.

ALVES-MAZZOTTI, Alda Judith. A "revisão da bibliografia" em teses e dissertações: meus tipos inesquecíveis - o retorno. In: BIANCHETTI; MACHADO (org.). A bússola do escrever: desafios e estratégias na orientação e escrita de teses e dissertações. 2. ed. Florianópolis: Editora da UFSC; São Paulo: Cortez, 2006. p. 25-44.

APIO, Adriana Linhares; SILVINO, Alexandre Magno Dias. A aula pública como instrumento preditor de desempenho docente no ensino superior. Avaliação, Campinas; Sorocaba, v. 18, n. 2, p. 277-298, jul. 2013. Doi: http://dx.doi.org/10.1590/S141440772013000200003. Disponível em: http://www.scielo.br/scielo.php?script=sci_arttext\&pid=S1414$40772013000200003 \& \operatorname{lng}=$ pt\&tlng=pt. Acesso em: 10 maio 2017. 
BIANCHETTI, Lucidio; MACHADO, Ana Maria Netto. A Bússola do escrever: desafios e estratégias na orientação e escritas de teses e dissertações. Florianópolis: Editora da UFSC; São Paulo: Cortez, 2006.

BISPO, Marcelo de Souza; COSTA, Francisco José da. Artigos como avaliação discente em disciplinas de pós-graduação: instrumento educativo ou subsistema de linha de montagem? Cad. EBAPE.BR, Rio de Janeiro, v. 14, n. 4, p. 1001-1010, dez. 2016. Doi: http://dx.doi.org/10.1590/1679-395141927. Disponível em: http://www.scielo.br/scielo.php?script=sci_arttext\&pid=S1679$39512016000401001 \& \operatorname{lng}=$ pt\&tlng=pt. Acesso em: 10 maio 2017.

CALVOSA, Marcello Vinicius Doria; REPOSSI, Melina Garcia; CASTRO, Pedro Marcos Roma de. Avaliação de resultados da capacitação docente: o pós-doutorado na Universidade Federal Fluminense sob a ótica da produção científica e bibliográfica. Avaliação, Campinas; Sorocaba, v. 16, n. 1, p. 99-122, mar. 2011 . Doi: http://dx.doi.org/10.1590/S141440772011000100006. Disponível em: http://www.scielo.br/scielo.php?script=sci_arttext\&pid=S1414$40772011000100006 \& \operatorname{lng}=$ pt\&tlng=pt. Acesso em: 10 maio 2017.

CANHADA, Diego Iturriet Dias; BULGACOV, Sergio. Strategic social practices and academic results: a doctorate in management at USP and UFRGS. Revista de Administração Pública, v. 45, n. 1, p. 7-32, 2011. Doi: https://dx.doi.org/10.1590/S0034-

76122011000100002. Disponível em:

http://www.scielo.br/scielo.php?script=sci_arttext\&pid=S0034$76122011000100002 \& \operatorname{lng}=$ pt\&tlng=pt. Acesso em: 14 abril 2017.

CAPES - COORDENAÇÃO DE APERFEIÇOAMENTO DE NÍVEL SUPERIOR. Disponível em: http://www.periodicos.capes.gov.br/. Acesso em: 12 abr. 2017.

CASTRO, Pedro Marcos Roma de; PORTO, Geciane Silveira. Evaluation of Outputs of Postdoctoral Research: brief notes about the scientific production in journals. Ensaio, Rio de Janeiro, v. 20, n. 74, p. 51-72, 2012. Doi: https://dx.doi.org/10.1590/S010440362012000100004. Disponível em: http://www.scielo.br/scielo.php?script=sci_arttext\&pid=S010440362012000100004\&lng=pt\&tlng=pt. Acesso em: 2 fev. 2017.

CASTRO, Rosane Michelli de. O programa de pós-graduação em educação da UNESP de Marília: contribuições para uma agenda de discussões sobre aspectos da política de pósgraduação no Brasil. Avaliação, Campinas; Sorocaba, v. 16, n. 1, p. 183-200, março 2011. Dio: http://dx.doi.org/10.1590/S1414-40772011000100010. Disponível em: http://www.scielo.br/scielo.php?script=sci_arttext\&pid=S1414$40772011000100010 \& \operatorname{lng}=$ pt\&tlng=pt. Acesso em 10 maio 2017.

CIRANI, Claudia Brito Silva; SILVA, Heloísa Helena Marques da; CAMPANARIO, Milton de Abreu. The evolution of brazilian graduate business administration programs. Revista de Administração Contemporânea, v. 16, n. 6, p. 765-805, 2012. Doi: https://dx.doi.org/10.1590/S1415-65552012000600002. Disponível em: http://www.scielo.br/scielo.php?script=sci_arttext\&pid=S141565552012000600002\&lng=pt\&tlng=pt. Acesso em: 12 jan. 2017.

CÓRDOVA, R.; GUSSO, D. A.; LUNA, S. V. A pós-graduação na América Latina: o caso brasileiro. Santa Maria: Editora da Universidade de S. Maria, 1986.

DRAKE, Miriam A. Encyclopedia of library and information science. New York, N.Y.: Marcel Dekker, 2004. 
DIAS, Evandro Dotto; RORATO, Rodrigo. Economic evolutionism in the brazilian graduate studies: an analysis from the vantage point of education. Avaliação, Campinas; Sorocaba, v. 19, n. 1, p. 193-226, 2014. Doi: https://dx.doi.org/10.1590/S1414-40772014000100010. Disponível em: http://www.scielo.br/scielo.php?script=sci_arttext\&pid=S141440772014000100010\&lng=pt\&tlng=pt. Acesso em: 12 mar. 2017. Quadros 2, 3 e 4.

EMBIRUÇU, Marcelo; FONTES, Cristiano; ALMEIDA, Luiz. Um indicador para a avaliação do desempenho docente em instituições de ensino superior. Ensaio, Rio de Janeiro, v. 18, n. 69, p. 795-820, dez. 2010. Doi: http://dx.doi.org/10.1590/S0104-40362010000400008.

Disponível em: http://www.scielo.br/scielo.php?script=sci_arttext\&pid=S1984$92302005000400008 \& \operatorname{lng}=$ pt\&tlng=pt. Acesso em: 10 maio 2017.

FERRARO, Alceu Ravanello. A ANPEd, a pós-graduação, a pesquisa e a veiculação da produção intelectual na área da educação. Revista Brasileira de Educação, Rio de Janeiro, v. 30, p. 47-69, 2005. Doi: https://dx.doi.org/10.1590/S1413-24782005000300005. Disponível em: http://www.scielo.br/scielo.php?script=sci_arttext\&pid=S1413$24782005000300005 \& \operatorname{lng}=$ t\&tlng=pt. Acesso em: 12 março 2017.

FESTINALLI, Rosane Calgaro. A formação de mestres em administração: por onde caminhamos? Organizações \& Sociedade, Salvador, v. 12, n. 35, p. 135-150, 2005. Doi: https://dx.doi.org/10.1590/S1984-92302005000400008. Disponível em: http://www.scielo.br/scielo.php?script=sci_arttext\&pid=S198492302005000400008\&lng=pt\&tlng=pt. Acesso em: 10 maio 2017.

FIALHO, Nadia Hage; HETKOWSKI, Tânia Maria. Mestrados Profissionais em Educação: novas perspectivas da pós-graduação no cenário brasileiro. Educ. rev., Curitiba, n. 63, p. 1934, mar. 2017. Doi: http://dx.doi.org/10.1590/0104-4060.49135. Disponível em: http://www.scielo.br/scielo.php?script=sci_arttext\&pid=S010440602017000100019\&lng=pt\&tlng=pt. Acesso em: 10 maio 2017.

FISCHER, Nilton Bueno. Comitê Científico da ANPEd: memórias, desafios e conquistas de uma ação construída em parcerias múltiplas. Rev. Bras. Educ., Rio de Janeiro, v. 12, n. 36, p. 410-423, dez. 2007. Doi: http://dx.doi.org/10.1590/S1413-24782007000300003. Disponível em: http://www.scielo.br/scielo.php?script=sci_arttext\&pid=S1413$24782007000300003 \& \operatorname{lng}=$ pt\&tlng=pt. Acesso em: 10 maio 2017.

FRANCISCO, Anete Maria et al. Avaliação da formação de enfermeiros: o reflexo dos métodos de ensino-aprendizagem e pressupostos curriculares na prática

profissional. Avaliação, Campinas; Sorocaba, v. 21, n. 2, p. 479-502, jul. 2016. Doi: http://dx.doi.org/10.1590/S1414-40772016000200009. Disponível em: http://www.scielo.br/scielo.php?script=sci_arttext\&pid=S1414$40772016000200479 \& \operatorname{lng}=$ pt\&tlng=pt. Acesso em: 10 maio 2017.

GODOI, Christiane Kleinübing; XAVIER, Wlamir Gonçalves. Productivism and its anomalies. Cadernos Ebape.br, Rio de Janeiro: v. 10, n. 2, p. 456-465, 2012.

GURGEL, Carmesina Ribeiro; LEITE, Raimundo Hélio. Avaliar aprendizagem: uma questão de formação docente. Ensaio, Rio de Janeiro, v. 15, n. 54, p. 145-168, 2007. Doi: https://dx.doi.org/10.1590/S0104-40362007000100009. Disponível em: http://www.scielo.br/scielo.php?script=sci_arttext\&pid=S0104$40362007000100009 \& \operatorname{lng}=$ pt\&tlng=pt. Acesso em: 14 fev. 2017.

HORTA, José Silvério Baía; MORAES, Maria Célia Marcondes de. O sistema CAPES de avaliação da pós-graduação: da área de educação à grande área de ciências humanas. Revista Brasileira de Educação, Rio de Janeiro, v. 30, n. 4, p. 95-116, 2005. Doi: 
https://dx.doi.org/10.1590/S1413-24782005000300008. Disponível em:

http://www.scielo.br/scielo.php?script=sci_arttext\&pid=S1413-

$24782005000300008 \& \operatorname{lng}=$ pt\&tlng=pt. Acesso em: 20 mar. 2017.

HORTALE, Virginia Alonso; KOIFMAN, Lilian. Programas de pós-graduação em Saúde Pública na Argentina e no Brasil: origens históricas e tendências recentes de processos de avaliação de qualidade. Interface-Comunicação, Saúde, Educação, Botucatu, v. 11, n. 21, p. 119-130, 2007. Doi: https://dx.doi.org/10.1590/S1414-32832007000100011. Disponível em: http://www.scielo.br/scielo.php?script=sci_arttext\&pid=S141432832007000100011\&lng=pt\&tlng=pt. Acesso em: 14 janeiro 2017.

HOSTINS, Regina Célia Linhares. Formação de pesquisadores em programas de excelência de pós-graduação em educação. Rev. Bras. Educ., Rio de Janeiro, v. 18, n. 53, p. 415-434, jun. 2013. Doi: http://dx.doi.org/10.1590/S1413-24782013000200010. Disponível em: http://www.scielo.br/scielo.php?script=sci_arttext\&pid=S1413-

$24782013000200010 \& \operatorname{lng}=$ pt\&tlng=pt. Acesso em: 10 maio 2017.

IKEDA, Ana Akemi; BACELLAR, Fátima Cristina Trindade. Revelando e compreendendo o relacionamento professor-aluno em Marketing. RAM, Rev. Adm. Mackenzie, São Paulo, v. 9, n. 5, p. 137-154, 2008. Doi: http://dx.doi.org/10.1590/S1678-69712008000500007. Disponível em: http://www.scielo.br/scielo.php?script=sci_arttext\&pid=S167869712008000500007\&lng=pt\&nrm=iso\&tlng=pt. Acesso em: 10 maio 2017.

KUENZER, Acacia Zeneida; MORAES, Maria Célia Marcondes de. Temas e tramas na pósgraduação em educação. Educ. Soc., Campinas, v. 26, n. 93, p. 1341-1362, dez. 2005. Doi: http://dx.doi.org/10.1590/S0101-73302005000400015. Disponível em:

http://www.scielo.br/scielo.php?script=sci_arttext\&pid=S0101$73302005000400015 \& \operatorname{lng}=$ pt\&tlng=pt. Acesso em: 10 maio 2017.

KUHLMANN JR., Moysés. Produtivismo acadêmico, publicação em periódicos e qualidade das pesquisas. Cad. Pesqui., São Paulo, v. 45, n. 158, p. 838-855, dez. 2015. Doi: http://dx.doi.org/10.1590/198053143597. Disponível em: http://www.scielo.br/scielo.php?script=sci_arttext\&pid=S010015742015000400838\&lng=pt\&tlng=pt. Acesso em: 10 maio 2017.

LÜDKE, Menga. Influências cruzadas na constituição e na expansão do sistema de pósgraduação stricto sensu em educação no Brasil. Revista Brasileira de Educação, v. 30, p. 117-123, 2005. Doi: https://dx.doi.org/10.1590/S1413-24782005000300009. Disponível em: http://www.scielo.br/scielo.php?script=sci_arttext\&pid=S1413$24782005000300009 \& \operatorname{lng}=$ pt\&tlng=pt. Acesso em: 23 dez. 2016.

MACCARI, Emerson Antonio; ALMEIDA, M.; NISHIMURA, A.; RODRIGUES, L. A gestão dos programas de pós-graduação em administração com base no sistema de avaliação da Capes. REGE. Revista de Gestão, São Paulo, v. 16, n. 4, p. 1-16, 1 dez. 2009.

DOI: https://doi.org/10.5700/issn.2177-8736.rege.2009.36682. Disponível em: https://www.revistas.usp.br/rege/article/view/36682. Acesso em 20 de dez.2016.

MACCARI, Emerson Antonio; RICCIO, Edson Luiz; MARTINS, Cibele Barsalini. The influence of the aacsb evaluation system on the management of graduate programs in the american business field. REAd. Revista Eletrônica de Administração, Porto Alegre, v. 19, n. 3, p. 738-766, 2013.

MACCARI, Emerson Antonio; NISHIMURA, Augusto Takerissa. Povoamento dos estratos conceitos 6 e 7 no sistema de avaliação da Capes pela área de administração, ciências contábeis e turismo nas avaliações trienais 2010 e 2013. REAd. Rev. eletrôn. adm., Porto 
Alegre, v. 20, n. 3, p. 601-624, dez. 2014. Doi: http://dx.doi.org/10.1590/1413-

2311.0442014.50134. Disponível em:

http://www.scielo.br/scielo.php?script=sci_arttext\&pid=S1413-

$23112014000300601 \& \operatorname{lng}=$ pt\&tlng=pt. Acesso em: 10 maio 2017.

MACCARI, E. A.; ALMEIDA, M. I. R.; RICCIO, E. L.; ALEJANDRO, T. B. Proposta de um modelo de gestão de programas de pós-graduação na área de Administração a partir dos sistemas de avaliação do Brasil (CAPES) e dos Estados Unidos (AACSB). Revista de

Administração, São Paulo, v. 49, n. 2, p. 369-383, 2014. Doi: http://dx.doi.org 10.5700/rausp1152 Disponível em: http://www.scielo.br/scielo.php?pid=S008021072014000200012\&script=sci_abstract\&tlng=pt. Acesso em 20 mar. 2017.

MACEDO, Elizabeth. Cultura performativa e pesquisa em educação: desafios para a ação política. Cad. Pesqui., São Paulo, v. 45, n. 158, p. 752-774, dez. 2015. Doi:

http://dx.doi.org/10.1590/198053143285. Disponível em:

http://www.scielo.br/scielo.php?script=sci_arttext\&pid=S0100-

15742015000400752\&lng=pt\&tlng=pt. Acesso em: 10 maio 2017.

MACEDO, Elizabeth; SOUSA, Clarilza Prado de. A pesquisa em educação no Brasil. Rev.

Bras. Educ., Rio de Janeiro, v. 15, n. 43, p. 166-176, abr. 2010. Doi:

http://dx.doi.org/10.1590/S1413-24782010000100012. Disponível em:

http://www.scielo.br/scielo.php?script=sci_arttext\&pid=S1413-

$24782010000100012 \& \operatorname{lng}=$ pt\&tlng=pt. Acesso em: 10 maio 2017.

MACHADO, Ana Maria Netto; BIANCHETTI, Lucídio. (Des)fetichização do produtivismo acadêmico: desafios para o trabalhador-pesquisador. Rev. adm. empres., São Paulo, v. 51, n. 3, p. 244-254, jun. 2011. Doi: http://dx.doi.org/10.1590/S0034-75902011000300005.

Disponível em: http://www.scielo.br/scielo.php?script=sci_arttext\&pid=S0034-

$75902011000300005 \& \operatorname{lng}=$ pt\&tlng=pt. Acesso em: 10 maio 2017.

MACHADO-DA-SILVA, Clóvis L. Respostas estratégicas da administração e contabilidade ao sistema de avaliação da capes. Organ. Soc., Salvador, v. 10, n. 28, p. 63-82, dez. 2003. Doi: http://dx.doi.org/10.1590/S1984-92302003000400005. Disponível em: http://dx.doi.org/10.1590/S1984-92302003000400005. Acesso em: 10 maio 2017.

MARIN, Maria José Sanches et al. Pós-graduação multiprofissional em saúde: resultados de experiências utilizando metodologias ativas. Interface (Botucatu), Botucatu, v. 14, n. 33, p. 331-344, jun. 2010. Doi: http://dx.doi.org/10.1590/S1414-32832010000200008. Disponível em: http://dx.doi.org/10.1590/S1414-32832010000200008. Acesso em: 10 maio 2017.

MARQUEZINE, Maria Cristina; TRAMONTINA, Viviane Maroneis. Produção científica dos alunos egressos do curso de especialização da Universidade Estadual de Londrina. Rev. bras. educ. espec, p. 101-122, 2006. Doi: https://dx.doi.org/10.1590/S1413-65382006000100008. Disponível em: http://www.scielo.br/scielo.php?script=sci_arttext\&pid=S1413$65382006000100008 \& \operatorname{lng}=$ pt\&tlng=pt. Acesso em: 20 jan. 2017.

MARTINS, Angela Maria; SOUSA, Sandra Zákia. A produção científica sobre avaliação educacional e gestão de sistemas e de escolas: o campo da questão entre 2000 e 2008. Ensaio: aval. pol. públ. educ., Rio de Janeiro, v. 20, n. 74, p. 9-26, mar. 2012. Doi:

http://dx.doi.org/10.1590/S0104-40362012000100002. Disponível em: http://www.scielo.br/scielo.php?script=sci_arttext\&pid=S0104-

$40362012000100002 \& \operatorname{lng}=$ pt\&tlng=pt. Acesso em: 10 maio 2017.

MELLO, Cristiane Marques de; CRUBELLATE, João Marcelo; ROSSONI, Luciano. Redes de coautorias entre docentes de programas brasileiros de pós-graduação (Stricto Sensu) em 
Administração: aspectos estruturais e dinâmica de relacionamento. RAM, Rev. Adm.

Mackenzie (Online), São Paulo, v. 10, n. 5, p. 130-153, out. 2009. Doi:

http://dx.doi.org/10.1590/S1678-69712009000500007. Disponível em:

http://www.scielo.br/scielo.php?script=sci_arttext\&pid=S1678-

$69712009000500007 \& \operatorname{lng}=$ pt\&tlng=pt. Acesso em: 10 maio 2017.

MELLO, Cristiane Marques de; CRUBELLATE, João Marcelo; ROSSONI, Luciano. Dinâmica de relacionamento e prováveis respostas estratégicas de programas brasileiros de pós-graduação em administração à avaliação da Capes: proposições institucionais a partir da análise de redes de co-autorias. Rev. adm. contemp., Curitiba, v. 14, n. 3, p. 434-457, 2010. Doi: http://dx.doi.org/10.1590/S1415-65552010000300004.

Disponível em: http://www.scielo.br/pdf/rac/v14n3/v14n3a04.pdf. Acesso em 10 maio de 2017.

MOREIRA, Antonio Flávio. A cultura da performatividade e a avaliação da pós-graduação em educação no Brasil. Educação em Revista, Belo Horizonte, v. 25, n. 3, p. 23-42, 2009. Doi: https://dx.doi.org/10.1590/S0102-46982009000300003. Disponível em:

http://www.scielo.br/scielo.php?script=sci_arttext\&pid=S0102-

46982009000300003\&lng=pt\&tlng=pt Acesso em: 23 fev. 2017.

MOREIRA, Maria Lígia; VELHO, Lea. Pós-graduação no Brasil: da concepção "ofertista linear" para "novos modos de produção do conhecimento" implicações para avaliação. Avaliação, Campinas; Sorocaba, v. 13, n. 3, p. 625-645, nov. 2008. Doi: http://dx.doi.org/10.1590/S1414-40772008000300002. Disponível em:

http://www.scielo.br/scielo.php?script=sci_arttext\&pid=S1414$40772008000300002 \& \operatorname{lng}=$ pt\&tlng=pt. Acesso em: 10 maio 2017.

MOREIRA, Maria Lígia; VELHO, Léa. Tracking alumni of the National Institute of Space Research (INPE): a tool for program evaluation. Avaliação, Campinas; Sorocaba, v. 17, n. 1, p. 255-288, 2012. Doi: https://dx.doi.org/10.1590/S1414-40772012000100013. Disponível em: http://www.scielo.br/scielo.php?script=sci_arttext\&pid=S1414$40772008000300002 \& \operatorname{lng}=$ pt\&tlng=pt. Acesso em: 5 jan. 2017.

MUÑOZ, Juan M. Escudero; ALONSO, Felipe Trillo. Un análisis crítico del Espacio Europeo de Educación Superior como reforma de la enseñanza universitaria: los programas Verifica y Docentia en el contexto español. Educ. rev., Curitiba, n. 57, p. 81-97, set. 2015. Doi: http://dx.doi.org/10.1590/0104-4060.42112. Disponível em: http://www.scielo.br/scielo.php?script=sci_arttext\&pid=S0104$40602015000300081 \& \operatorname{lng}=$ es\&tlng=es. Acesso em: 10 maio 2017.

NOSELLA, Paolo. Research in education: a balance of the production of postgraduate programmes in education. Revista Brasileira de Educação, Rio de Janeiro, v. 15, n. 43, p. 177-183, 2010. Doi: https://dx.doi.org/10.1590/S1413-24782010000100013. Disponível em: http://www.scielo.br/scielo.php?script=sci_arttext\&pid=S1413-

$24782010000100013 \& \operatorname{lng}=$ pt\&tlng=pt. Acesso em 1 maio 2017.

PATRUS, Roberto; DANTAS, Douglas Cabral; SHIGAKI, Helena Belintani. O produtivismo acadêmico e seus impactos na pós-graduação stricto sensu: uma ameaça à solidariedade entre pares?. Cad. EBAPE.BR, Rio de Janeiro, v. 13, n. 1, p. 1-18, mar. 2015. Doi:

http://dx.doi.org/10.1590/1679-39518866. Disponível em:

http://www.scielo.br/scielo.php?script=sci_arttext\&pid=S1679-

39512015000100002\&lng=pt\&tlng=pt. Acesso em: 10 maio 2017.

PEIXOTO, Maria do Carmo de Lacerda. Avaliação institucional externa no SINAES: considerações sobre a prática recente. Avaliação, Campinas; Sorocaba, v. 16, n. 1, p. 11-36, 
mar. 2011. Doi: http://dx.doi.org/10.1590/S1414-40772011000100002. Disponível em: http://www.scielo.br/scielo.php?script=sci_arttext\&pid=S1414-

$40772011000100002 \& \operatorname{lng}=$ pt\&tlng=pt. Acesso em: 10 maio 2017.

PEZZI, Silvana; STEIL, Andrea Valéria. Análise do processo de exame de grau na pósgraduação stricto sensu. Educ. Pesqui., São Paulo, v. 35, n. 1, p. 33-50, abr. 2009. Doi: http://dx.doi.org/10.1590/S1517-97022009000100003. Disponível em:

http://www.scielo.br/scielo.php?script=sci_arttext\&pid=S1517-

97022009000100003\&lng=pt\&tlng=pt. Acesso em: 10 maio 2017.

PUCCI, Bruno. Fórum dos Coordenadores de Programas de Pós-Graduação em Educação: apontamentos históricos. Rev. Bras. Educ., Rio de Janeiro, v. 12, n. 36, p. 424-442, dez. 2007. Doi: http://dx.doi.org/10.1590/S1413-24782007000300004. Disponível em: http://www.scielo.br/scielo.php?script=sci_arttext\&pid=S1413-

$24782007000300004 \& \operatorname{lng}=$ pt\&tlng=pt. Acesso em: 10 maio 2017.

RAMALHO, Betania Leite; MADEIRA, Vicente de Paulo Carvalho. A pós-graduação em educação no Norte e Nordeste: desafios, avanços e perspectivas. Revista Brasileira de Educação, Rio de Janeiro, v. 30, p. 70-81, 2005. Doi: https://dx.doi.org/10.1590/S141324782005000300006. Disponível em: http://www.scielo.br/scielo.php?script=sci_arttext\&pid=S1413$24782005000300006 \& \operatorname{lng}=$ pt\&tlng=pt. Acesso em: 28 mar. 2017.

RAMOS, Milena Yumi; VELHO, Lea. Doctoral education in Brazil: the weariness of the current model in face of the challenges posed by the emergence of the global system of science. Avaliação, Campinas; Sorocaba, v. 18, n. 1, p. 219-246, 2013. Doi: https://dx.doi.org/10.1590/S1414-40772013000100012. Disponível em : http://www.scielo.br/scielo.php?script=sci_arttext\&pid=S1414$40772013000100012 \& \operatorname{lng}=$ pt\&tlng=pt. Acesso em: 14 fev. 2017.

REGO, Teresa Cristina. Produtivismo, pesquisa e comunicação científica: entre o veneno e o remédio. Educ. Pesqui., São Paulo, v. 40, n. 2, p. 325-346, jun. 2014. Doi: http://dx.doi.org/10.1590/S1517-97022014061843. Disponível em: http://www.scielo.br/scielo.php?script=sci_arttext\&pid=S151797022014000200003\&lng=pt\&tlng=pt. Acesso em: 10 maio 2017.

ROBLEDO, Sebastian; OSORIO, German; LOPEZ, Carolina. Networking en pequeña empresa: una revisión bibliográfica utilizando la teoria de grafos. Revista Vínculos, Manizales, Colômbia, v. 11, n. 2, p. 6-16, 2015.

ROCHA, Nívea Maria Fraga. Auto-avaliação de centros de pós-graduação: uma proposta em ação. Ensaio: aval.pol.públ.Educ., Rio de Janeiro, v. 14, n. 53, p. 487-506, dez. 2006. Doi: http://dx.doi.org/10.1590/S0104-40362006000400006. Disponível em:

http://www.scielo.br/scielo.php?script=sci_arttext\&pid=S0104-

$40362006000400006 \& \operatorname{lng}=$ pt\&tlng=pt. Acesso em: 10 maio 2017.

ROSA, Soraya Diniz; LOPES, Roseli Esquerdo. Tecendo os fios entre educação e saúde: avaliação do Programa da Residência Multiprofissional em Saúde. Avaliação, Campinas; Sorocaba, v. 21, n. 2, p. 637-660, jul. 2016. Doi: http://dx.doi.org/10.1590/S141440772016000200016. Disponível em: http://www.scielo.br/scielo.php?script=sci_arttext\&pid=S1414$40772016000200637 \& \operatorname{lng}=$ pt\&tlng=pt. Acesso em: 10 maio 2017. 
RÖSSING, Tânia Marta Kuchenbecker. Se não lêem ou lêem pouco, como esperar que escrevam? In: BIANCHETTI, Lucídio (org). Trama e texto: leitura crítica, escrita criativa. Passo Fundo: Ed. UPF, 2002.

RAUEN, Margarida Gandara; FILHO, Afonso Figueiredo. A educação internacional e os resultados de cooperação Brasil-Alemanha na Unicentro. Avaliação, Campinas; Sorocaba, v. 21, n. 3, p. 673-690, nov. 2016. Doi: http://dx.doi.org/10.1590/S1414-40772016000300002. Disponível em: http://www.scielo.br/scielo.php?script=sci_arttext\&pid=S1414$40772016000300673 \& \operatorname{lng}=$ pt\&tlng=pt. Acesso em: 10 maio 2017.

SANCHES, Otavio Prospero; SANCHES, Lucia Helena Aponi. O desafio da avaliação de equipes de alta capacitação e autonomia: o caso do credenciamento de docentes na pósgraduação. Avaliação, Campinas; Sorocaba, v. 16, n. 1, p. 123-147, mar. 2011. Doi: http://dx.doi.org/10.1590/S1414-40772011000100007. Disponível em:

http://www.scielo.br/scielo.php?script=sci_arttext\&pid=S1414$40772011000100007 \& \operatorname{lng}=$ pt\&tlng=pt. Acesso em: 10 maio 2017.

SILVA, Talita Fernanda da; NAKANO, Tatiana de Cássia. Criatividade no contexto educacional: análise de publicações periódicas e trabalhos de pós-graduação na área da psicologia. Educação e Pesquisa: Revista da Faculdade de Educação da Universidade de São Paulo, São Paulo, v. 38, n. 3, p. 743-759, 2012. Doi: http://dx.doi.org/10.1590/S151797022012005000013. Disponível em: http://www.scielo.br/scielo.php?pid=S151797022012000300014\&script=sci_abstract\&tlng=pt. Acesso em: 2 maio 2017.

SINAY, Maria Cristina Fogliatti de et al. Environmental management education \& research in Brazilian graduate courses of business administration. RAM. Revista de Administração Mackenzie,São Paulo, v. 14, n. 3, p. 55-82, 2013. Doi: https://dx.doi.org/10.1590/S167869712013000300004. Disponível em:

http://www.scielo.br/scielo.php?script=sci_arttext\&pid=S1678$69712013000300004 \& \operatorname{lng}=$ pt\&tlng=pt. Acesso em: 2 maio 2017.

SOBRAL, Fernanda A. da Fonseca. Qualidade acadêmica e relevância social e econômica da educação superior. Cad. CEDES, Campinas, v. 29, n. 78, p. 227-241, ago. 2009. Doi: http://dx.doi.org/10.1590/S0101-32622009000200006. Disponível em: http://www.scielo.br/scielo.php?script=sci_arttext\&pid=S010132622009000200006\&lng=pt\&tlng=pt. Acesso em: 10 maio 2017.

SOUZA, Saulo Aparecido; REINERT, José Nilson. Student participation in the publications of graduate schools: a comparative study between public and private administration programs. Avaliação, Campinas; Sorocaba, v. 17, n. 2, p. 481-502, 2012. Doi: https://dx.doi.org/10.1590/S1414-40772012000200010. Disponível em: http://www.scielo.br/scielo.php?script=sci_arttext\&pid=S1414$40772012000200010 \& \operatorname{lng}=$ pt\&tlng=pt. Acesso em: 20 abr. 2017.

TREE of Science. 2017. Disponível em: http://tos.manizales.unal.edu.co/about/. Acesso em: 15 abr. 2017.

TREVISAN, Amarildo Luiz; DEVECHI, Catia Piccolo Viero; DIAS, Evandro Dotto. Avaliação da avaliação da pós-graduação em educação do Brasil: quanta verdade é suportável? Avaliação, Campinas; Sorocaba, v. 18, n. 2, p. 373-392, jul. 2013. Doi: http://dx.doi.org/10.1590/S1414-40772013000200008. Disponível em: http://www.scielo.br/scielo.php?script=sci_arttext\&pid=S1414-

$40772013000200008 \& \operatorname{lng}=$ pt\&tlng=pt. Acesso em: 10 maio 2017. 
VELLOSO, Jacques. Mestres e doutores no país: destinos profissionais e políticas de pósgraduação. Cad. Pesqui., São Paulo, v. 34, n. 123, p. 583-611, dez. 2004. Doi:

http://dx.doi.org/10.1590/S0100-15742004000300005. Disponível em:

http://www.scielo.br/scielo.php?script=sci_arttext\&pid=S0100-

15742004000300005\&lng=pt\&tlng=pt. Acesso em: 10 maio 2017.

VERHINE, Robert Evan. Pós-graduação no Brasil e nos Estados Unidos: uma análise comparativa. Educação, Porto Alegre, v. 31, n. 2, p. 166-172, 2008.

VIANNA, William Barbosa; ENSSLIN, Leonardo; GIFFHORN, Edilson. A integração sistêmica entre pós-graduação e educação básica no Brasil: contribuição teórica para um "estado da arte". Ensaio, Rio de Janeiro, v. 19, n. 71, p. 327-344, 2011. Disponível em: http://www.scielo.br/scielo.php?script=sci_arttext\&pid=S141440772013000100010\&lng=pt\&tlng=pt Acesso em: 8 maio 2017.

VIDOR, Gabriel; FOGLIATTO, Flávio Sanson; CATEN, Carla ten. Development of an instrument to evaluate graduate programs. Avaliação, Campinas; Sorocaba, v. 18, n. 1, p. 181-199, 2013.Doi: https://dx.doi.org/10.1590/S1414-40772013000100010 . Disponível em: http://www.scielo.br/pdf/ensaio/v19n71/a06v19n71.pdf. Acesso em: 20 dez. 2017.

VILAÇA, Murilo Mariano; PALMA, Alexandre. Comentários sobre avaliação, pressão por publicação, produtivismo acadêmico e ética científica. Cad. Pesqui., São Paulo, v. 45, n. 158, p. 794-816, dez. 2015. Doi: http://dx.doi.org/10.1590/198053142836. Disponível em: http://www.scielo.br/scielo.php?script=sci_arttext\&pid=S010015742015000400794\&lng=pt\&tlng=pt. Acesso em: 10 maio 2017.

WERLE, Flávia Obino Corrêa. Panorama das políticas públicas na educação brasileira: uma análise das avaliações externas de sistemas de ensino. Revista Lusófona de

Educação,Lisboa, n. 27, p. 159-179, 2014.

WERLE, Flávia Obino Corrêa. Políticas de avaliação em larga escala na educação básica: do controle de resultados à intervenção nos processos de operacionalização do ensino. Ensaio, Rio de Janeiro, v. 19, n. 73, p. 769-792, dez. 2011. Doi: http://dx.doi.org/10.1590/S010440362011000500003. Disponível em:

http://www.scielo.br/scielo.php?script=sci_arttext\&pid=S0104-

$40362011000500003 \& \operatorname{lng}=$ pt\&tlng=pt. Acesso em: 10 maio 2017.

WEB of Science. 2017. Disponível em: https://webofknowledge.com. Acesso em: 10 abr. 2017.

WOOD JR., Thomaz; COSTA, Caio César. Avaliação do impacto da produção científica de programas selecionados de pós-graduação em Administração por meio do índice H. Revista de Administração, São Paulo, v. 50, n. 3, p. 325-337, 2015. 